generated: (1) Satisfaction with current care, (2) Key timepoints for improved cancer care, (3) Information and research. Satisfaction with current care amongst patients was high in spite of reported high levels of unmet needs on the SCNS indicating low expectation. Most patients undergoing cancer treatment were unsure when they should access primary care. Patients and carers highly valued proactive contact from their practice following diagnosis although this was perceived as unusual. Post-oncology discharge was a key time-point for information needs and support by patients and clinicians. Patients were reluctant to consider palliative care in spite of palliative care needs - indicating low understanding. Some felt therapeutic benefit from completing study measures. In spite of positive attitudes to cancer research by consented participants, some had poor understanding of study procedures.

Conclusion Patients are satisfied with cancer primary care despite unmet needs. Palliative care is poorly understood by many who may benefit. Research participation may benefit some patients.

\section{IS A DEMENTIA NURSE SPECIALIST A FEASIBLE AND ACCEPTABLE WAY TO IMPROVE CARE TOWARDS END OF LIFE IN DEMENTIA?}

Marie Poole, Claire Bamford, Emma McLellan, Dorothy Coe, Susan Hrisos, Louise Robinson, on behalf of the SEED team. Newcastle University

\subsection{6/bmjspcare-2019-ASP.14}

Background End of life care for people with dementia is recognised as complex and often suboptimal. The SEED project (Supporting Excellence in End of Life Care) is a 5 year NIHR programme grant aimed at improving end of life care for people with dementia. Through research with key stakeholder groups, an intervention comprising of a Dementia Nurse Specialist (DNS) and supportive resources was developed. The DNS role was implemented for 12 months, with data collected alongside to assess the feasibility and acceptability of the role.

Methods A mixed methods approach was used to explore feasibility and acceptability. We focus on the qualitative process evaluation, which explored the views and experiences of: people with dementia; family carers; palliative specialists; mental health specialists; GPs; primary care specialists, care home staff; and the Dementia Nurse Specialists, through interview and observations

Results The role of a Dementia Nurse Specialist to improve care in advancing dementia was considered to be feasible and acceptable by all stakeholder groups. Key benefits identified were: the value of the role being based in primary care; the DNS role fits with existing services; the value of a specialist role to coordinate multidisciplinary care in dementia towards end of life; and the opportunities to build relationships with families, to facilitate advance planning conversations and provide timely support when needed. Additional factors were identified around the support needed to deliver the role and the key skills required.

Conclusion The DNS role was considered as feasible and acceptable as a way of improving the delivery of care towards end of life for people with dementia and their families. However, issues remain around the most effective way to implement the role in a context of scarce resources.
Free Papers 16 - 18 | Service Development II

\section{INTEGRATED ONCOLOGY AND PALLIATIVE CARE: ANALYSIS OF A NEW 'TRIGGERS' SERVICE FOR LUNG CANCER PATIENTS}

J Droney, Y Kano, J Nevin, L Kamal, A Kennett, R Oloko, S Popat, T Wiseman, M Kabir, M Perkins, I Rathwell, J Wood, A Stevens. Royal Marsden NHS Foundation Trust

\subsection{6/bmjspcare-2019-ASP.15}

Background Global leading cancer organisations recommend that palliative care should be available to all patients, regardless of stage of disease. We established a novel Integrated Palliative Care service ('Triggers' Service) for lung cancer outpatients. New patients are screened by oncologists using a brief palliative care referral 'Triggers' tool to identify patients who should be referred for full assessment. Assessment of palliative care need is completed using validated tools (IPOS Integrated Palliative Outcome Scale, Performance status).

Aims Evaluation of the feasibility and effectiveness of the 'Triggers' service using data collected from the initial 12 months of the service.

Methods Prospective observational service evaluation, using descriptive statistical analyses. Approved by the local Committee for Clinical Research

Results 496 eligible patients were identified, of which 433 (87\%) were reviewed through the 'Triggers' service within 8 weeks of initial oncology outpatient clinic consultation.

$62 \%$ (272) of patients were positive for at least one Palliative Care referral 'Trigger' tool item. IPOS and performance status data were available for 269 of these. 79\% (212/269) patients had at least 1 'severe' or 'overwhelming' palliative care need. 76\% (205/269) patients were performance status 0-1.

At time of analysis, 120 patients had died. The median (range) time between earliest palliative care review and death/ censor date was 197 days (range 3-485 days), compared to 79 (10-1315) days at baseline before establishment of 'Triggers' service. As a result of this service, the number of new patients reviewed annually by the hospital Palliative Care team increased by $30 \%$.

Conclusion/discussion The 'Triggers service appears feasible and effective in establishing early palliative care for patients with lung cancer. Further evaluation and economic modelling is required to facilitate roll out to other tumour groups and services.

\section{CARING FOR THE PALLIATIVE MEDICINE MULTIDISCIPLINARY TEAM: PILOTING AN EMOTIONAL SUPPORT FORUM}

Virginia Bray, Heather Davies, Sarah Malyon, Mandy Scott, Caroline Stancer, Patrick Costello. Nottingham University Hospitals NHS Trust

\subsection{6/bmjspcare-2019-ASP.16}

Background Working in palliative medicine requires emotional resilience and is associated with compassion fatigue and burnout. However, research into support strategies for palliative care teams is lacking. Schwartz rounds improve caregiver psychological wellbeing but are limited by implementation costs. This project aimed to: ascertain staff satisfaction with their counselling service; measure wellbeing; and pilot an emotional support forum. 
Methods This study was conducted in a specialist palliative care unit with 20 inpatient beds and day therapy unit. Discussions were held with the counselling, medical and nursing staff to develop the support service. A survey was distributed to MDT members, giving 24 anonymous responses. Subsequently, a monthly emotional support forum was piloted, facilitated by a ward nurse and supported by the counsellors and ward manager. A follow-up survey was distributed 16 months later with 32 respondents.

Results The initial survey identified staff may benefit from emotional support, with over 50\% experiencing disturbed sleep, intrusive thoughts and feeling sad or depressed at some point over the past month. Preferred options were mindfulness sessions and an emotional support forum. Over $70 \%$ of respondents to the second survey attended the forum at some point. Of those, $85 \%$ felt it an inclusive and safe environment and $74 \%$ found it helpful. $48 \%$ could not attend as often as hoped, predominantly due to time constraints and staffing. There were no significant differences between measures of anxiety and depression before and after forum introduction.

Conclusions Staff were more aware of the counselling service to support patients/relatives than themselves, but many engaged with the forum and found it helpful. Areas for improvement include increasing awareness and facilitating forum attendance. This study was not designed to detect objective changes in anxiety/depression, however, our qualitative findings support the forum as a low cost and convenient approach to improving staff wellbeing.

\section{HEART FAILURE AND PALLIATIVE CARE: AN INTEGRATED SERVICE FOR PATIENTS ACROSS HOSPITAL AND COMMUNITY SETTINGS}

JR Ross, F Hodson, S Karwatowski, M Albarjas, R Nash, J Smith, R George. St Christopher's Hospice, King's College Hospital, Princess Royal University Hospital

\subsection{6/bmjspcare-2019-ASP.17}

Background An integrated clinical service for patients with end-stage heart failure (HF) was funded by Bromley Clinical Commissioning Group; Bromley has no community HF nurse specialists.

Method Six GP practices participated and referral criteria were agreed. Patients were assessed and followed up by an Advanced Nurse Practitioner, with experience in HF and palliative care (PC), who worked closely with cardiology consultants and HF/PC hospital teams, the Community PC Team (St Christopher's Hospice) and primary care colleagues. Following initial assessment, patients were discussed at joint MDMs to assess both cardiac and palliative needs. We report data from the service evaluation of this clinical pilot.

Results 102 patients, mean age 84.2 \pm 10.2 years, were referred over 1 year. 89 were accepted. 55 (61.8\%) had LVSD, 14 (15.7\%) patients had HF with preserved ejection fraction, 13 (14.6\%) had predominately valvular dysfunction, 5 (5.6\%) had right sided HF. Patients had multiple co-morbidities, significant renal impairment and mental health diagnoses (depression and dementia). Two thirds required considerable assistance or were immobile $>50 \%$ of the time, with $18 \%$ predominately bedbound. 29 (33\%) lived alone, 49 (55\%) with family, and 12\% in care homes. The majority of carers were female (86\%), $25 \%$ of carers frequently/nearly always 'did not have time for themselves' with $40 \%$ reporting a significant impact of caring on their own health. Meta-Analysis Global Group in Chronic Heart Failure (MAGGIC) scores were not accurate in predicting outcome. $44 \%$ of patients died during the pilot. Of these, $69 \%$ died at home/hospice $(41 \% / 28 \%)$ versus $31 \%$ in hospital. Further PROMs including integrated palliative care outcome scale (iPOS) will be presented.

Conclusion This pilot was positive and demonstrates benefits and challenges of joint working between integrated teams. We will discuss how ongoing integrated practice can be developed locally with dissemination of good practice and joint working to other groups.

\section{Free Papers $19-21$ | Training}

\section{DO PALLIATIVE CARE CHAMPIONS INCREASE STAFF CONFIDENCE TO HAVE DIFFICULT CONVERSATIONS?}

Jane Manson, Helen Blomfield, Louise Patterson, Angela Todd, Lisa Farndon. Sheffield Teaching Hospitals

\subsection{6/bmjspcare-2019-ASP.18}

Background Previous research and recent media coverage have identified that healthcare professionals who don't work in a palliative care setting do not feel comfortable talking to patients about palliative-related issues. As caseloads become more frail and complex, there is an increasing need for staff members at all levels to feel more confident discussing issues such as advanced life planning, deteriorating function, and future care needs. Champions have proven to be highly effective in knowledge translation in order to instigate positive changes in healthcare, however there is no evidence evaluating the 'champion approach' in palliative care or in a rehabilitation setting.

Aim To evaluate the introduction of the palliative care champion role on staff confidence and patient outcomes.

Methods A cohort study of four bed-based intermediate care units ( 2 intervention, 2 control) was used. Three champions based in the two intervention units received specialist training and disseminated this to the remaining cohort. This was followed by a 5 month data collection period.

Results Confidence of the intervention unit staff increased an average of 1.9 points on a scale of $1-10$ post training $(0.1$ control). This confidence continued to increase to an average of 2.4 points following the 5 month intervention period $(0.3$ control). There was no change to perceived contact with palliative patients or barriers to conversation.

Conclusion Introducing palliative care champions could be an easy, cost-effective way of increasing staff confidence to have difficult conversations with patients.

\section{TO EXPLORE THE UTILITY OF THE BREATHING, THINKING, FUNCTIONING (BTF) CLINICAL MODEL IN MANAGING BREATHLESSNESS THROUGH A PRACTICAL WORKSHOP AIMING TO EQUIP PROFESSIONALS}

Declan Cawley, Aileen McCartney, Anna Spathis. Wisdom Hospice, University of Cambridge

\subsection{6/bmjspcare-2019-ASP.19}

Background Breathlessness is a common and distressing symptom within progressive respiratory conditions. Emerging research advocates that irrespective of diagnosis, management 